International Mathematical Forum, Vol. 8, 2013, no. 33, 1615 - 1620

HIKARI Ltd, www.m-hikari.com

http://dx.doi.org/10.12988/imf.2013.38165

\title{
Green's Relations on the Menger Algebra of n-ary Ordered Preserving Operations
}

\author{
Siwanaph Samartkoon ${ }^{1}$ \\ Department of Mathematics, Faculty of Science \\ Khon kaen University, Khon kaen 40002, Thailand \\ samartkoon@hotmail.com \\ Prakit Jampachon \\ Department of Mathematics, Faculty of Science \\ Khon kaen University, Khon kaen 40002, Thailand \\ prajam@kku.ac.th
}

Copyright (c) 2013 Siwanaph Samartkoon and Prakit Jampachon. This is an open access article distributed under the Creative Commons Attribution License, which permits unrestricted use, distribution, and reproduction in any medium, provided the original work is properly cited.

\section{Introduction}

For integer $n \geq 1$, let $O^{n}(A)$ be the set of all $n$-ary operations defined on a set $A$ and let $O(A):=\bigcup_{n \geq 1} O^{n}(A)$ be the set of all operations defined on $A$. For $f \in O^{n}(A)$ and $g_{1}, \ldots, g_{n} \in O^{m}(A)$, we define the superposition operation $S_{m}^{n}: O^{n}(A) \times\left(O^{m}(A)\right)^{n} \longrightarrow O^{m}(A)$ by

$$
S_{m}^{n}\left(f, g_{1}, \ldots, g_{n}\right)\left(a_{1}, \ldots, a_{m}\right):=f\left(g_{1}\left(a_{1}, \ldots, a_{m}\right), \ldots, g_{n}\left(a_{1}, \ldots, a_{m}\right)\right)
$$

for all $a_{1}, \ldots, a_{m} \in A$. If $n=m$, we will write for short $S^{n}$ instead of $S_{n}^{n}$. For each $n \geq 1$ and each $1 \leq j \leq n$, the $n-$ ary function $e_{j}^{n}: A^{n} \longrightarrow A$ defined on A by $e_{j}^{n}\left(a_{1}, \ldots, a_{n}\right):=a_{j}$ is called the $j-t h$ projection mapping of arity $n$.

In [2] and also [3], an algebra $\mathcal{M}:=\left(M ; S^{n}, e_{1}, \ldots, e_{n}\right)$ of type $\tau=$ $(n+1,0, \ldots, 0)$ is called a unitary Menger algebra of rank $n$ if it satisfies the following axioms: 
(C1) $\tilde{S}^{n}\left(X_{0}, \tilde{S}^{n}\left(Y_{1}, X_{1}, \ldots, X_{n}\right), \ldots, \tilde{S}^{n}\left(Y_{n}, X_{1}, \ldots, X_{n}\right)\right)$

$\left.\approx \tilde{S}^{n}\left(\tilde{S}^{n}\left(X_{0}, Y_{1}, \ldots, Y_{n}\right), X_{1}, \ldots, X_{n}\right)\right)$

(C2) $\tilde{S}^{n}\left(\lambda_{j}, X_{1}, \ldots, X_{n}\right) \approx X_{j} \quad$ for all $i \leq j \leq n$,

(C3) $\tilde{S}^{n}\left(X_{j}, \lambda_{1}, \ldots, \lambda_{n}\right) \approx X_{j} \quad$ for all $i \leq j \leq n$.

(Here $\tilde{S}^{n}$ is an $(n+1)$ - ary operation symbol, $\lambda_{1}, \ldots, \lambda_{n}$ are nullary operation symbols and $X_{0}, X_{1}, \ldots, X_{n}, Y_{1}, \ldots, Y_{n}$ are variables.)

In [3], an algebra $\left(M ; S^{n}\right)$ of type $(n+1)$ which satisfies $(C 1)$ is called a Menger algebra of rank $n$.

Let $(A, \leq)$ be a finite chain. We define the relation $\leq^{(n)}$ on $A^{n}$ by $\left(a_{1}, \ldots, a_{n}\right) \leq^{(n)}$ $\left(b_{1}, \ldots, b_{n}\right)$ if $a_{i} \leq b_{i}$ for all $i=1, \ldots, n$ and define the set

$$
\operatorname{Pol}_{\leq}^{(n)}(A):=\left\{f \in O^{n}(A) \mid f \text { preserves } \leq\right\},
$$

where $f$ preserves $\leq$ means if $\left(a_{1}, \ldots, a_{n}\right) \leq^{(n)}\left(b_{1}, \ldots, b_{n}\right)$ then $f\left(a_{1}, \ldots, a_{n}\right) \leq$ $f\left(b_{1}, \ldots, b_{n}\right)$. We obtain $\left(\operatorname{Pol}_{\leq}^{(n)}(A) ; S^{n}\right)$ is a Menger algebra of rank $n$.

In [3], for Menger algebras of rank $n$, Green' s relations were defined as the followings:

Definition 1.1 Let $\left(M ; S^{n}\right)$ be a Menger algebra of rank $n$ and let $a, b \in M$.

(i) $a \mathcal{L} b$ if either $a=b$ or there are elements $s_{1}, \ldots, s_{n}, t_{1}, \ldots, t_{n} \in M$ such that $S^{n}\left(a, s_{1}, \ldots, s_{n}\right)=b$ and $S^{n}\left(b, t_{1}, \ldots, t_{n}\right)=a$.

(ii) $a \mathcal{R} b$ if either $a=b$ or there are elements $s, t \in M$ such that $S^{n}(s, a, \ldots, a)=b$ and $S^{n}(t, b, b, \ldots, b)=a$.

(iii) $\mathcal{D}=\mathcal{R} \circ \mathcal{L}(=\mathcal{L} \circ \mathcal{R})$.

(iv) $\mathcal{H}=\mathcal{R} \cap \mathcal{L}$

(v) $a \mathcal{J} b$ if either $a=b$ or there are elements $s, s_{1}, \ldots, s_{n} \in M$ with $a=$ $S^{n}\left(s, s_{1}, \ldots, s_{n}\right)$ such that at least one of the factors is equal to $b$ and there are elements $t, t_{1}, \ldots, t_{n} \in M$ with $b=S^{n}\left(t, t_{1}, \ldots, t_{n}\right)$ such that at least one of the factors is equal to a.

\section{The main results}

In this section we will study on the Menger algebra $\left(\operatorname{Pol}_{\leq}^{(n)}(A) ; S^{n}\right)$ where $A$ is a finite chain. For simplification we use $\underline{a}$ instead of $\left(a_{1}, a_{2}, \ldots, a_{n}\right)$. We also 
use $\widehat{a}$ for the $n$-tuple consisting of the same element $a \in A$ i.e. $\widehat{a}=(a, a, \ldots, a)$. For each $f \in \operatorname{Pol}_{\leq}^{(n)}(A)$, let

$$
\operatorname{Kerf}:=\left\{(\underline{a}, \underline{b}) \in A^{n} \times A^{n} \mid f(\underline{a})=f(\underline{b})\right\},
$$

and

$$
\operatorname{Imf}:=\left\{x \in A \mid \exists \underline{a} \in A^{n}, f(\underline{a})=x\right\} .
$$

Let $x \in \operatorname{Im} f$ we set $f^{-1}(x)=\left\{\underline{a} \in A^{n} \mid f(\underline{a})=x\right\}$.

For a subset $B \subseteq A^{n}$ we define the set $f(B):=\{f(\underline{a}) \in A \mid \underline{a} \in B\}$. For any two subsets $B, B^{\prime}$ of the chain $A$, we write $B \leq B^{\prime}$ if $b \leq b^{\prime}$ for all $b \in B, b^{\prime} \in B^{\prime}$. Let $\triangle_{\text {Imf }}=\left\{\widehat{x} \in A^{n} \mid x \in \operatorname{Imf}\right\}$.

In a Menger algebra $\left(\operatorname{Pol}_{\leq}^{(n)}(A) ; S^{n}\right)$, we obtain the following theorems.

Theorem 2.1 Let $f, g \in \operatorname{Pol}_{\leq}^{(n)}(A)$. Then $f \mathcal{R} g$ if and only if the following conditions are satisfy

(i) $\operatorname{Kerf}=\operatorname{Kerg}$,

(ii) for each $x, y \in \operatorname{Imf}, x \leq y$ iff $g\left(f^{-1}(x)\right) \leq g\left(f^{-1}(y)\right)$.

Proof. Suppose that $f \mathcal{R} g$. There exist $\alpha, \beta \in \operatorname{Pol}_{\leq}^{(n)}(A)$ such that $f=S^{n}(\alpha, g, \ldots, g)$ and $g=S^{n}(\beta, f, \ldots, f)$. Let $(\underline{a}, \underline{b}) \in \operatorname{Ker} f$. We have $f(\underline{a})=f(\underline{b})$ and hence $\widehat{f(\underline{a})}=\widehat{f(\underline{b})}$. Thus $g(\underline{a})=S^{n}(\beta, f, \ldots, f)(\underline{a})=\beta(\widehat{f(\underline{a})})=\beta(\widehat{f(\underline{b})})=S^{n}(\beta, f, \ldots, f)(\underline{b})=$ $g(\underline{b})$. That is $K e r f \subseteq K e r g$. Similarly, we get $K \operatorname{Ker} g \subseteq K \operatorname{Kerf}$. Thus $K \operatorname{Kerf}=$ $\operatorname{Kerg}$. Let $x, y \in \operatorname{Imf}$ such that $x \leq y$. Then there exist $\underline{a}, \underline{b} \in A^{n}$ such that $f(\underline{a})=x$ and $f(\underline{b})=y$ (i.e, $\underline{a} \in f^{-1}(x)$ and $\left.\underline{b} \in f^{-1}(y)\right)$. We have $g(\underline{a})=$ $S^{n}(\beta, f, \ldots, f)(\underline{a})=\beta(\widehat{f(\underline{a})})=\beta(\widehat{x}) \leq \beta(\widehat{y})=\beta(\widehat{f(\underline{b})})=S^{n}(\beta, f, \ldots, f)(\underline{b})=$ $g(\underline{b})$. Therefore, $g\left(f^{-1}(x)\right) \leq g\left(f^{-1}(y)\right)$. In the same way, for all $x, y \in \operatorname{Im} f$, if $g\left(f^{-1}(x)\right) \leq g\left(f^{-1}(y)\right)$ we have $x \leq y$.

Conversely, suppose that $K \operatorname{erf}=\operatorname{Kerg}$ and for each $x, y \in \operatorname{Imf}$, we have $x \leq y$ iff $g\left(f^{-1}(x)\right) \leq g\left(f^{-1}(y)\right)$. For each $x \in \operatorname{Im} f$, we choose one element $\underline{a_{x}} \in f^{-1}(x)$. Define an $n-$ ary operation $\beta: A^{n} \longrightarrow A$ by $\beta(\widehat{x})=g\left(\underline{a_{x}}\right)$ for all $x \in \operatorname{Imf}$ and $\beta(\underline{c})=c_{0}$ for all $\underline{c} \in A^{n} \backslash \triangle_{\text {Imf }}$ and $c_{0}$ is a fixed element in $A$. Since $K \operatorname{erf}=K \operatorname{erg}$, we have $f(\underline{a})=f(\underline{b})$ iff $g(\underline{a})=g(\underline{b})$. Thus $\beta$ is well-defined. Let $\underline{a} \in A^{n}$ such that $f(\underline{a})=x$. We have $S^{n}(\beta, f, \ldots, f)(\underline{a})=$ $\beta(f, \ldots, f)(\underline{a})=\beta(\widehat{f(\underline{a})})=\beta(\widehat{x})=g\left(\underline{a_{x}}\right)=g(\underline{a})$. That is $g=S^{n}(\beta, f, \ldots, f)$. Let $x, y \in \operatorname{Imf}$ such that $x \leq y$. By assumption, we have $g\left(\underline{a_{x}}\right) \leq g\left(a_{y}\right)$. Thus $\beta(\widehat{x}) \leq \beta(\widehat{y})$. For all $\underline{a}, \underline{b} \in A^{n} \backslash \triangle_{\text {Imf }}$ such that $\underline{a} \leq \underline{b}$, we have $\beta(\underline{a})=\overline{c_{0}}=\beta(\underline{b})$ and hence $\beta \in \operatorname{Pol}_{\leq}^{(n)}(A)$. Similarly, we can get well-defined an $n$-ary ordered preserving operation $\alpha$ such that $f=S^{n}(\alpha, g, \ldots, g)$. Therefore, $f \mathcal{R} g$.

For Green's relation $\mathcal{L}$, we obtain the following result. 
Theorem 2.2 Let $f, g \in \operatorname{Pol}_{\leq}^{(n)}(A)$. Then $f \mathcal{L} g$ if and only if the following conditions are satisfy;

(i) $\operatorname{Imf}=\operatorname{Img}$.

(ii) For each $x, y \in \operatorname{Imf}$ such that $x \leq y$. Then

there exist $\underline{a} \in f^{-1}(x), \underline{b} \in f^{-1}(y)$ such that $\underline{a} \leq \underline{b} \quad$ iff there exist $\underline{a^{\prime}} \in g^{-1}(x), \underline{b^{\prime}} \in g^{-1}(y)$ such that $\underline{a^{\prime}} \leq \underline{b^{\prime}}$.

Proof. Suppose that $f \mathcal{L} g$. Then there exist $s_{1}, \ldots, s_{n}, t_{1}, \ldots, t_{n} \in \operatorname{Pol}_{\leq}^{(n)}(A)$ such that $f=S^{n}\left(g, s_{1}, \ldots, s_{n}\right)$ and $g=S^{n}\left(f, t_{1}, \ldots, t_{n}\right)$. Let $x \in \operatorname{Imf}$. Then $f(\underline{a})=x$ for some $\underline{a} \in A^{n}$. We have $x=f(\underline{a})=S^{n}\left(g, s_{1}, \ldots, s_{n}\right)(\underline{a})=$ $g\left(s_{1}(\underline{a}), \ldots, s_{n}(\underline{a})\right) \in \operatorname{Img}$. Thus $\operatorname{Imf} \subseteq \operatorname{Img}$. Similarly, we have Img $\subseteq$ $\operatorname{Imf}$ and hence $\operatorname{Imf}=\operatorname{Img}$. Let $x, y \in \operatorname{Imf}, x \leq y$. If there exists $\underline{a} \in$ $f^{-1}(x), \underline{b} \in f^{-1}(y)$ such that $\underline{a} \leq \underline{b}$, we have $x=f(\underline{a})=S^{n}\left(g, s_{1}, \ldots, s_{n}\right)(\underline{a})=$ $g\left(s_{1}(\underline{a}), \ldots, s_{n}(\underline{a})\right)=g\left(\underline{a}^{\prime}\right)$,

and $y=f(\underline{b})=S^{n}\left(g, s_{1}, \ldots, s_{n}\right)(\underline{b})=g\left(s_{1}(\underline{b}), \ldots, s_{n}(\underline{b})\right)=g\left(\underline{b^{\prime}}\right)$ where $\underline{a^{\prime}}=$ $\left(s_{1}(\underline{a}), \ldots, s_{n}(\underline{a})\right) \in g^{-1}(x)$ and $\underline{b^{\prime}}=\left(s_{1}(\underline{b}), \ldots, s_{n}(\underline{b})\right) \in g^{-1}(y)$. Since $s_{1}, \ldots s_{n} \in$ $\operatorname{Pol}_{\leq}^{n}(A)$ and $\underline{a} \leq \underline{b}$, we have $s_{i}(\underline{a}) \leq s_{i}(\underline{b})$ for all $i=1,2, \ldots, n$. Thus $\underline{a^{\prime}}=\left(s_{1}(\underline{a}), \ldots, s_{n}(\underline{a})\right) \leq\left(s_{1}(\underline{b}), \ldots, s_{n}(\underline{b})\right)=\underline{b^{\prime}}$. Similarly, we get that if there exist $\underline{a^{\prime}} \in g^{-1}(x), \underline{b^{\prime}} \in g^{-1}(y)$ such that $\underline{a^{\prime}}<\underline{b^{\prime}}$ then we have there exist $\underline{a} \in f^{-1}(x), \underline{b} \in f^{-1}(y)$ such that $\underline{a} \leq \underline{b}$.

Conversely, suppose that the conditions $(i)$ and $(i i)$ are hold. For each $x, y \in$ $\operatorname{Im} f$ such that $x \leq y$, we choose one element $a_{x}^{*} \in g^{-1}(x)$ satisfies the following conditions;

-if no elements $\underline{a} \in f^{-1}(x), \underline{b} \in f^{-1}(y)$ such that $\underline{a} \leq \underline{b}$, we choose $a_{x}^{*}=$ $\left(a_{x 1}^{*}, \ldots, a_{x n}^{*}\right) \in g^{-1}(x)$, and $a_{y}^{*}=\left(a_{y 1}^{*}, \ldots, a_{y n}^{*}\right) \in g^{-1}(y)$

-if there exist elements $\underline{a} \bar{\in} f^{-1}(x), \underline{b} \in f^{-1}(y)$ such that $\underline{a} \leq \underline{b}$, there exist $\underline{a^{\prime}} \in g^{-1}(x), \underline{b^{\prime}} \in g^{-1}(y)$ such that $\underline{a^{\prime}} \leq \underline{b^{\prime}}$, we choose $\underline{a_{x}^{*}}=\underline{a^{\prime}}=\left(a_{x 1}^{*}, \ldots, a_{x n}^{*}\right) \in$ $g^{-1}(x)$ and $\underline{a_{y}^{*}}=\underline{b^{\prime}}=\left(a_{y 1}^{*}, \ldots, a_{y n}^{*}\right) \in g^{-1}(y)$. Since $A^{n}=\bigcup_{x \in I m f} f^{-1}(x)$ is a disjoint union, then for all $j=1, \ldots, n$, we define $n$-ary operations $s_{j}: A^{n} \longrightarrow A$ by $s_{j}(\underline{a})=a_{x j}^{*}$ for all $\underline{a} \in f^{-1}(x)$ and for all $x \in I m f$.

Let $\underline{a}, \underline{b} \in A^{n}$ such that $\underline{a} \leq \underline{b}$. We have

-if $\underline{a}, \underline{b} \in f^{-1}(x)$ then $s_{j}(\underline{a})=a_{x j}^{*}=s_{j}(\underline{b})$,

-if $\underline{a} \in f^{-1}(x), \underline{b} \in f^{-1}(y)$ such that $\underline{a} \leq \underline{b}$ then $s_{j}(\underline{a})=a_{x j}^{*} \leq a_{y j}^{*}=s_{j}(\underline{b})$ and hence $s_{j} \in \operatorname{Pol}_{\leq}^{n}(A)$. Let $\underline{a} \in A^{n}$ such that $f(\underline{a})=x$. We have $S^{n}\left(g, s_{1}, \ldots, s_{n}\right)(\underline{a})=\bar{g}\left(s_{1}(\underline{a}), \ldots, s_{n}(\underline{a})\right)=g\left(a_{x 1}^{*}, \ldots, a_{x n}^{*}\right)=g\left(\underline{a_{x}^{*}}\right)=x=f(a)$. Thus $f=S^{n}\left(g, s_{1}, \ldots, s_{n}\right)$. Similarly, we can get well-defined $n$-ary operations $t_{k}: A^{n} \longrightarrow A$ for all $k=1, \ldots, n$ such that $g=S^{n}\left(f, t_{1}, \ldots, t_{n}\right)$.

Therefore, $f \mathcal{L} g$.

For Green's relation $\mathcal{H}$, we obtain the following : 
Theorem 2.3 Let $f, g \in \operatorname{Pol}_{\leq}^{(n)}(A)$. Then $f \mathcal{H} g$ if and only if $f=g$.

Proof. If $f=g$, we have $f \mathcal{R} g$ and $f \mathcal{L} g$. Hence, $f \mathcal{H} g$.

Suppose that $f \mathcal{H} g$, then $f \mathcal{R} g$ and $f \mathcal{L} g$. Thus Kerf $=K \operatorname{Ker} g$ and $I m f=I m g$. If $|\operatorname{Imf}|=1$, we have $f=g$. We assume that $|\operatorname{Im} f| \geq 2$ and $f \neq g$. Then there exist $x \in \operatorname{Im} f$ such that $f^{-1}(x) \neq g^{-1}(x)$. Say that $x_{0}$ is a smallest element of $\operatorname{Im} f$ such that $f^{-1}\left(x_{0}\right) \neq g^{-1}\left(x_{0}\right)$. Then there exist $y, z \in \operatorname{Im} f$ such that $x_{0} \leq y, x_{0} \leq z$ and $g^{-1}\left(x_{0}\right)=f^{-1}(y), f^{-1}\left(x_{0}\right)=g^{-1}(z)$. Since $f \mathcal{R} g$, we have $g\left(f^{-1}\left(x_{0}\right)\right) \leq g\left(f^{-1}(y)\right)$. But $g\left(f^{-1}\left(x_{0}\right)\right)=\{z\} \geq\left\{x_{0}\right\}=g\left(f^{-1}(y)\right)$ gives a contradiction. Thus $f^{-1}(x)=g^{-1}(x)$ for all $x \in \operatorname{Im} f=\operatorname{Img}$. Hence, $f=g$.

For Green's relation $\mathcal{D}$, we obtain the following:

Theorem 2.4 Let $f, g \in \operatorname{Pol}_{\leq}^{(n)}(A)$. Then $f \mathcal{D} g$ if and only if the following conditions are satisfy:

(i) $|\operatorname{Imf}|=|\operatorname{Img}|$.

(ii) For Imf $=\left\{x_{1}, x_{2}, \ldots, x_{r}\right\}$, Img $=\left\{y_{1}, y_{2}, \ldots, y_{r}\right\}$ where $x_{1}<x_{2}<\cdots<$ $x_{r}$ and $y_{1}<y_{2}<\cdots<y_{r}$, we have for each $x_{i}, x_{j}$ there exist $\underline{a} \in$ $f^{-1}\left(x_{i}\right), \underline{b} \in f^{-1}\left(x_{j}\right)$ such that $\underline{a} \leq \underline{b} \quad$ iff there exist $\underline{a^{\prime}} \in g^{-1}\left(y_{i}\right), \underline{b^{\prime}} \in$ $g^{-1}\left(y_{j}\right)$ such that $\underline{a}^{\prime} \leq \underline{b}^{\prime}$.

Proof. Suppose that $f \mathcal{D} g$, then there exist $\lambda \in \operatorname{Pol}_{\leq}^{(n)}(A)$ such that $f \mathcal{R} \lambda$ and $\lambda \mathcal{L} g$. We have $\operatorname{Kerf}=\operatorname{Ker} \lambda$ and $\operatorname{Im} \lambda=\operatorname{Img}$ and so $|\operatorname{Imf}|=|\operatorname{Im} \lambda|=|\operatorname{Img}|$. Let Imf $=\left\{x_{1}, x_{2}, \ldots, x_{r}\right\}, \operatorname{Img}=\left\{y_{1}, y_{2}, \ldots, y_{r}\right\}$ where $x_{1}<x_{2}<\cdots<x_{r}$ and $y_{1}<y_{2}<\cdots<y_{r}$. Since $\lambda \mathcal{L} g$, we have for all $y_{i} \leq y_{j}$ there exist $\underline{a} \in \lambda^{-1}\left(y_{i}\right)$, $\underline{b} \in \lambda^{-1}\left(y_{j}\right)$ such that $\underline{a} \leq \underline{b}$ iff there exist $\underline{a^{\prime}} \in g^{-1}\left(y_{i}\right), \underline{b^{\prime}} \in g^{-1}\left(y_{j}\right)$ such that $\underline{a^{\prime}} \leq \underline{b^{\prime}}$. Since $f \mathcal{R} \lambda$, we have $f^{-1}\left(x_{i}\right)=\lambda^{-1}\left(y_{i}\right)$ for all $i=1, \ldots, r$. Therefore, for all $x_{i} \leq x_{j}$ there exist $\underline{a} \in f^{-1}\left(x_{i}\right), \underline{b} \in f^{-1}\left(x_{j}\right)$ such that $\underline{a} \leq \underline{b}$ iff there exist $\underline{a^{\prime}} \in g^{-1}\left(y_{i}\right), \underline{b^{\prime}} \in g^{-1}\left(y_{j}\right)$ such that $\underline{a^{\prime}} \leq \underline{b^{\prime}}$

Conversely, suppose that the conditions $(i)$ and $(i i)$ are hold. Let $\operatorname{Imf}=$ $\left\{x_{1}, x_{2}, \ldots, x_{r}\right\}, \operatorname{Img}=\left\{y_{1}, y_{2}, \ldots, y_{r}\right\}$ where $x_{1}<x_{2}<\cdots<x_{r}$ and $y_{1}<y_{2}<$ $\cdots<y_{r}$. Since $A^{n}=\bigcup_{x \in \operatorname{Imf}} f^{-1}(x)$ is a disjoint union, then for each $x_{i} \in \operatorname{Imf}$, we defined an $n$-ary operation $\lambda: A^{n} \longrightarrow A$ by $\lambda(\underline{a})=y_{i}$ for all $\underline{a} \in f^{-1}\left(x_{i}\right)$. We have $\operatorname{Ker} f=\operatorname{Ker} \lambda$ and $\lambda\left(f^{-1}\left(x_{i}\right)\right)=\left\{y_{i}\right\} \leq\left\{y_{j}\right\}=\lambda\left(f^{-1}\left(x_{j}\right)\right)$ iff $x_{i} \leq x_{j}$. That is $f \mathcal{R} \lambda$. Since $|\operatorname{Im} f|=|\operatorname{Img}|$, we have $\operatorname{Img}=\operatorname{Im} \lambda$. By assumption (ii) we have, for all $x_{i} \leq x_{j}$ there exist $\underline{a} \in \lambda^{-1}\left(y_{i}\right)=f^{-1}\left(x_{i}\right), \underline{b} \in \lambda^{-1}\left(y_{j}\right)=$ $f^{-1}\left(x_{j}\right)$ such that $\underline{a} \leq \underline{b}$ iff there exist $\underline{a^{\prime}} \in g^{-1}\left(y_{i}\right), \underline{b^{\prime}} \in g^{-1}\left(y_{j}\right)$ such that $\underline{a^{\prime}}<\underline{b^{\prime}}$. That is $\lambda \mathcal{L} g$. Therefore, $f \mathcal{D} g$.

For Green's relation $\mathcal{J}$, we we obtain the following: 
Theorem 2.5 Let $f, g \in \operatorname{Pol}_{\leq}^{(n)}(A)$ such that $\mathcal{J}=\operatorname{Pol}_{\leq}^{(n)}(A) \times \operatorname{Pol}_{\leq}^{(n)}(A)$.

Proof. Since the projections $e_{j}^{n} \in \operatorname{Pol}_{\leq}^{(n)}(A)$ for all $j=1,2, \ldots, n$, then $f=S^{n}\left(e_{1}^{n}, f, g, \ldots, g\right)$ and $g=S^{n}\left(e_{1}^{n}, g, f, \ldots, f\right)$. This means that $f \mathcal{J} g$ for all $f, g \in \operatorname{Pol}_{\leq}^{(n)}(A)$. Hence, $\mathcal{J}=\operatorname{Pol}_{\leq}^{(n)}(A) \times \operatorname{Pol}_{\leq}^{(n)}(A)$.

\section{Acknowledgment}

This research is supported by the Centre of Excellence in Mathematics, the commission on Higher Education, Ministry of Education,Thailand.

\section{References}

[1] J.M. Howie, Fundamentals of Semigroup Theory, Oxford Science Publication, Clarendon Press, Oxford 1995.

[2] B. Schein and V.S. Trochimenko, Algebras of multiplace functions, Semigroup Forum Vol. 17 (1979). 1-64.

[3] K. Denecke, P. Jampachon, Regular Elements and Green's relations in Menger Algebras of terms, Discussiones Mathematicae, General Algebra and Applications, 26(2006), 85-109.

Received: August 5, 2013 